\title{
TV/Series
}

Hors séries 1 | 2016

Lost: (re)garder l'île

\section{La promesse de Lost (À propos de la Saison 2)}

\section{Guillaume Dulong}

\section{(2) OpenEdition}

\section{Journals}

Electronic version

URL: http://journals.openedition.org/tvseries/1636

DOI: 10.4000/tvseries.1636

ISSN: 2266-0909

\section{Publisher}

GRIC - Groupe de recherche Identités et Cultures

\section{Electronic reference}

Guillaume Dulong, «La promesse de Lost (À propos de la Saison 2) », TV/Series [Online], Hors séries

1 | 2016, Online since 14 October 2016, connection on 20 April 2019. URL : http:// journals.openedition.org/tvseries/1636; DOI : 10.4000/tvseries.1636

This text was automatically generated on 20 April 2019.

\section{(c) (i) (9)}

TV/Series est mis à disposition selon les termes de la licence Creative Commons Attribution - Pas d'Utilisation Commerciale - Pas de Modification 4.0 International. 


\title{
La promesse de Lost (À propos de la Saison 2)
}

\author{
Guillaume Dulong
}

Je me vois comme un homme qui a donné sur mille écueils et qui, après avoir échappé de justesse à un naufrage, en se faufilant dans une passe, a cependant la témérité d'affronter la mer sur le même bateau battu par les tempêtes et faisant eau de toutes parts, et qui pousse même l'ambition à faire le tour du globe dans des conditions aussi désavantageuses. Le souvenir que j'ai de mes erreurs passées et de mes perplexités me fait douter de l'avenir. La misérable condition, la faiblesse, le désordre des facultés que je dois employer dans mes recherches accroissent mes craintes. Et l'impossibilité de me corriger ou de

corriger ces facultés me réduit presque au désespoir. Plutôt périr sur ce rocher ingrat où je me trouve, que m'aventurer sur cet océan sans limite qui fuit dans l'immensité1 !

1 Après le succès de la première saison de lost s'achevant sur l'explosion de la trappe, l'enjeu de l'écriture de la suite fut tel que Cuse et Lindelof racontent qu'Abrams fut le premier à leur demander ce qu'il y avait sous la trappe ${ }^{2}$. Ce problème, qui est le fil directeur de cet ensemble de 24 épisodes - voire de la série toute entière - fut résolu dès le premier épisode de la saison 2. Sous la trappe, il n'y a pas une autre trappe, il y a un homme, Desmond (Henry Ian Cusick). Et il a la clé. Celui-ci rentre les chiffres «maudits » 4815162342 et appuie sur le bouton Execute toutes les 108 minutes car « on » - l'occulte et tentaculaire Dharma Initiative - lui a dit que cela était nécessaire au salut du monde à la suite d'un « incident » dont la station est le sarcophage, « Namaste and good luck ». Est-ce 
une sordide plaisanterie ou est-ce vrai ? Le personnage de Lost est-il crédule et conditionné ou bien sait-il, voire veut-il, ce qu'il fait?

2 Ce premier épisode, "Man of science, Man of faith », est celui qui a réalisé la plus grosse audience de toute la série, à savoir 23.470 .000 spectateurs aux États-Unis. Néanmoins, c'est également après cette saison 2 que Lost ne sera plus diffusée en prime time en France. Cela est d'autant plus paradoxal qu'y sont présentés les termes d'un pacte de lecture avec les téléspectateurs. Les configurations métadiégétiques déployées dans ces épisodes, comme celle de la répétition, nous indiquent les règles du jeu de l'opération de communication qu'est Lost, offrant une réflexion sur la promesse qu'est l'acte de raconter et sur la manière de la comprendre. Dans ce cadre, la fin même du récit y est annoncée : dans l'épisode «The End» (S06E17), la question du bouton et de ce qui se passe si l'on n'appuie pas dessus divise toujours autant les « mêmes » personnages.

Dès lors, cette deuxième saison requiert une réception plus attentive, voire prospective, car la narration devient transmédiatique. En avril 2006, la Lost Experience, opération de marketing viral, reprend, par la variété de ces modes d'énonciation de récits parallèles à découvrir, le thème de l'énoncé, à savoir la société secrète et l'ésotérisme. La création d'un vrai site internet de l'entreprise fictive Hanso et la parution du roman Bad Twin dont l'auteur est le personnage Gary Troup ${ }^{3}$, mort sur le vol Oceanic 815, participent d'un processus d'immersion dans l'univers raconté par le truchement de la Dharma Initiative. Ces dispositifs permettent à des récepteurs littéralement religieux - religio dérivant du latin relegere, "relire attentivement", s'il faut en croire Cicéron ou Benveniste ${ }^{4}$ d'approfondir leur croyance en le monde du récit promis, en articulant les éléments en hypothèses, hypothèses systématisées en un quasi-savoir. Ce savoir les oppose alors aux néophytes et aux profanes : il leur permet de saisir un sens caché, d'anticiper certains événements du récit et d'en justifier certains retournements. Leur compréhension, leur croyance en l'unité et la singularité de l'univers du récit, est plus intégrale, moins superficielle, car constituée d'hypothèses et de théories partagées par une communauté d'initiés ${ }^{5}$. Corrélativement leurs attentes sont plus grandes... et peut-être mal placées dans le sens où l'on ferait crédit à un débiteur non solvable ${ }^{6}$.

4 La question posée de manière récurrente par le Lostie ultra-orthodoxe sur les forums de discussion en ligne, "Ont-'ils' prévu la fin? Tout est-il déjà écrit?", faisant de Cuse et Lindelof les maitres du Grand Orient Dharma, mène à la rage furieuse du 23 mai 2010 contre ces faux prophètes, sur ces mêmes forums. Reviennent alors les reproches à Abrams, et à l'écurie de scénaristes estampillés Bad Robot, d'être des teasers plus que des narrateurs faisant de leur intrigue un vaste MacGuffin leur permettant de ne satisfaire aucune promesse à la fin, tout en déployant des effets de pathos et autres effets paroxystiques pour la seule virtuosité des renversements de rapports amour/haine entre personnages. Ils sont comme les Autres, comme ce Grand Inquisiteur cynique de la légende d'Ivan dans Les Frères Karamazov qui entend dominer les hommes par le mystère, le miracle et l'autorité8. Pour autant, la série ne manque pas, surtout à travers le personnage de John Locke, de mettre en garde contre un délire de surinterprétation nécessairement décevant:dans la saison 2, la prolifération des chiffres et autres hiéroglyphes croît en proportion directe avec la paranoïa et le ressentiment des personnages.

5 À cet égard, Pacôme Thiellement remarque que le jeu de piste Dharma et sa symbolique cryptique nous présentent littéralement des stations, des étapes par lesquelles il est possible de formuler une interprétation du récit plus dense, érudite et cultivée. Cette 
intertextualité approfondit notre amour pour celui-ci comme pour les textes (romans, pièces de théâtres, mythes et écrits bibliques) auxquels il se réfère 9 . Par exemple, on découvre que le nombre 108 est l'addition de 4, 8, 15, 16, 23 et 42, qu'il renvoie aussi au nombre de grains du chapelet du Bouddha, mais aussi qu'il est la valeur numérique de la Géhenne selon la Kabbale, et cette enquête crée un espace d'échange commun entre les enquêteurs lostéens. Néanmoins, pour tirer profit de ces informations, la collecte ne suffit pas; il est indispensable de dégager une unité nouvelle expliquant la conjonction de ces références s'excluant apparemment l'une l'autre: comment se fait-il qu'un même dispositif soit désigné à la fois comme voie d'illumination et vallée de larmes?

6 Ces stations sont donc des points de passages, littéralement des étapes. D’ailleurs, par un mouvement dialectique, le cours de la narration les voue à la destruction, encourageant une réception dynamique. En d'autres termes, la compréhension de Lost devient un handicap si les différents niveaux sémantiques dégagés sont hypostasiés par le spectateur opposant un sens littéral superficiel à un sens figuré essentiel. Cela ne signifie pas qu'une telle ligne de partage est vaine, que celui qui hiérarchise les niveaux de sens du récit est nécessairement un intégriste, mais qu'en dernière instance, cette échelle de valeurs doit elle-même être évaluée. Sinon la lecture est statique, c'est-à-dire ésotérique et autoritaire, et d'enquêteur, le spectateur fidèle risque alors de se muer en inquisiteur.

Dans cette perspective, nous nous proposons d'étudier le traitement des thèmes de la foi et du sacrifice dans la saison 2. Par voie de réflexion, cela nous amènera à interroger le niveau de croyance à accorder au récit audiovisuel lui-même. En effet, notre hypothèse est que la promesse de récit de Lost prescrit une suspension volontaire d'incrédulité ${ }^{10}$ imitant l'opération du bunker: la narration répète sa promesse initiale (résoudre la catastrophe du crash sur l'île) si nous répétons notre assentiment, tout en prenant le risque de la déception produite par la répétition elle-même. Celle-ci accroît l'attente d'un dénouement à venir tout en semblant discréditer le contenu de l'histoire par un effet de « sur place » sui référentiel.

8 La réactivation toutes les 108 minutes d'un compteur dans la Station du Cygne a une fonction déclarée plutôt vraisemblable, compte tenu des codes des genres fantastique et de science-fiction vers lesquels tend cette saison. Il est donc plausible qu'en appuyant sur le bouton, on sauve le monde. Néanmoins, comme Locke l'exige de Jack, cette opération requiert notre bonne volonté tenant du sacrifice de soi. C'est une allégorie du double procès de lecture/relecture d'une série télévisée répondant à un double procès d'écriture/réécriture propre à ce type de récits. En effet, celle-ci doit jouer à la fois sur la répétition de situations familières et sur l'irruption d'événements et de renversements juste assez catastrophiques pour remettre les compteurs à zéro d'une saison (voire d'un épisode) à l'autre : climax, cliffhanger, anagnorisis... Comme le montre Umberto Eco, dans ses analyses des comics ou des feuilletons, ces techniques narratives font que la fiction sérielle, en dépit du postulat d'une chronologie globale reliant les épisodes, tend à se déployer dans une dimension de présent intransitif ${ }^{11}$. Ce présent se rapproche de la synchronie de l'analyse structurale, mais aussi de l'habitude, voire du déni nostalgique symptomatique d'un comportement névrotique et d'une procrastination posttraumatique. Peu à peu, chaque épisode a l'air de n'être la conséquence, ni la cause, d'aucun événement extérieur mais n'en continue pas moins à produire des effets d'échanges (plutôt que de progression) de situations, de caractères ou de pensées. Les retournements de situations, les twists, se déploient quasiment pour eux-mêmes faisant fi d'objets, voire de raisons, à propos desquels s'opposer ou s'unir. Ainsi, à la fin de « Man of 
science, Man of faith", est-on faiblement surpris de voir que l'homme qui parlait à Jack dans son flashback, est l'homme du souterrain. L'univers du récit consiste alors en un désordre homogène, ou un multivers monotone, plus qu'un ordre harmonisant des éléments hétérogènes ${ }^{12}$. Les sous promesses et sous reconnaissances des sous intrigues proposées se réitèrent à chaque épisode pour des personnages différents certes, mais selon des codes de composition semblables. Elles acquièrent alors dans ce cadre une fonction de confirmation aussi spectaculaire que prévisible. Cela nous rassure à court terme mais diffère le problème originel et en repousse l'échéance ultime aux calendes grecques. La fiction nourrit dès lors un angoissant oubli du long terme, c'est-à-dire une indétermination croissante de la place des péripéties relativement aux enjeux principaux et initiaux.

Cette écriture a quelque chose de vertigineux qui agace ceux qui veulent croire en l'unité d'une trame close et qui considèrent que les effets de pathos sont vulgaires au nom de la supposition d'un sens diachronique final définitif répondant à la promesse initiale. Ils ont en partie raison : le présent itératif des épisodes tient du déjà vu, du cliché, voire du temps perdu dans le sens proustien du terme, celui qu'on ne peut et qu'on ne veut pas vivre au présent dans sa pleine possibilité, mais que l'on se représente à partir de souvenirs passés déjà là ou de projets pas encore là, combinant juste ces mêmes souvenirs. Ainsi en va-t-il de ce que vivent les personnages de la série sur l'île. Cela n'est pas compris par eux au présent mais comme représentation, c'est-à-dire relativement à des flashbacks voire flashforwards ressemblants. De même pour nous, spectateur, ce présent habituel de la série est une représentation passée car nous le reconnaissons à partir de notre souvenir de la promesse de l'ouverture du récit et dans l'attente d'une fin qui lui aura attribué une signification dans un cours diachronique sensé, mais il s'agit une représentation passée qui ne dit pas son nom. Comme le déni, ce présent itératif prétend congédier le passage du temps (le début et la fin de l'histoire) tout en l'admettant implicitement. Dès lors par ce report, par ce divertissement de la question du passage, il augmente le contenu d'une promesse et d'une attente de fin qui en deviennent angoissantes et vertigineuses car objectivement intenables. Ce que Gabriel Bortzmeyer saisit admirablement en faisant de Lost une métasérie lorsqu'il écrit : « Tout était déjà dans Lost, scénario du surplace et de la survie par excellence, où seul l'espoir minimal fonde la possibilité du récit de sa déception. Gagner du temps, repousser l'échéance, c'est ce à quoi s'en tiennent les personnages, et, avec eux, la série ${ }^{13}$.»

10 Toutefois, s'agacer de ce vertige de l'écriture sérielle, comme le firent ceux qui lynchèrent Cuse et Lindelof de leur acrimonie numérique, ne fait que le renforcer, car c'est refuser d'admettre sa propre angoisse d'une fin. Or les auteurs n'ont pourtant jamais cessé d'annoncer la fin, quoiqu'elle ne soit pas seulement dans le récit mais qu'elle se révèle comme un appel à un au-delà du récit. Comprendre cet appel par «l'espoir minimal » permet de retrouver le temps perdu, voire de vraiment gagner le temps, au sens où Tarkovski, réalisateur du dostoïevskien voire prélostéen Stalker (1979) déclarait que voir un film c'est moins perdre son temps que le gagner car il change notre regard sur le monde. Ainsi, la réactivation du compteur évoque aussi le rapport affectif rituel et domestique qu'a le récepteur avec la série, mais aussi son rapport théorique de reprise des divers épisodes, nécessaire à la constitution d'un grand récit total, présumé, qui ne serait pas qu'un agrégat de coups de théâtre hétérogènes ${ }^{14}$. Or cette réactivation fait osciller les personnages de la série, comme les spectateurs, entre incrédulité et 
enthousiasme, au point de mener à cette issue paradoxale : il faut sacrifier la station pour la sauver ${ }^{15}$.

11 C'est dans cette perspective que la série se réfère explicitement au schème de l'« acte de foi ", du saut dans l'incertain, voire l'absurde. Certains personnages de Lost sont ainsi appelés à se perdre manifestement : ils assument une prise de risque extrême, optant pour l'alternative la plus improbable, sinon objectivement, au moins subjectivement. Ils initient un acte dont l'issue est possible, en théorie, mais dont l'occurrence, en fait, ne dépend pas d'eux, d'où l'évocation de l'opposition indécidable du hasard et du destin dans leur choix. Dans le même temps, ils sont résignés : ils sont convaincus de l'improbabilité non seulement de la réalisation de l'événement, mais aussi de la relation causale supposée entre l'action initiée et l'événement prévu. Enfin ils sont amenés à professer vouloir faire l'action et l'avoir librement décidée. Or cette démarche n'est pas sans évoquer une version radicale de la suspension volontaire d'incrédulité du récepteur de fiction; attitude requise, au moins tacitement, par le narrateur. Radicale car le narrateur sériel en multipliant les climax et le pathos jusqu'à l'improbable, paraît contrevenir aux règles génériques qu'il annonce, exigeant du spectateur une ferveur plus ultra.

d'opposer, d'un côté la croyance ordinaire, relativement justifiée, et, de l'autre, la foi. La première est intéressée et accordée après délibération, donc sous condition. La seconde est désintéressée et accordée avant toute justification rationnelle voire, pour les versions les plus radicales, en dépit de toute justification rationnelle. Toutefois, ce « saut » dans le pur possible est de l'ordre du vécu, d'un présent ouvert sur un futur incertain. En témoigner dans le récit est problématique car le récit imite et répète ; son temps est théoriquement le passé : on suppose que les auteurs rapportent, c'est-à-dire que la fin leur est déjà connue, et précède, en droit, le début pour le spectateur, cette supposition forgeant sa confiance en la promesse de récit. Donc l'expérience temporelle que fait le spectateur lorsqu'il anticipe les épisodes à venir est moins une ouverture au pur possible (quand bien même ce qui est raconté appartiendrait au genre du merveilleux) qu'une forme d'induction voire d'abduction de ce qu'ont probablement et vraisemblablement déjà pensé les auteurs.

13 Présenter ce " saut » en lui-même consisterait à se référer directement à l'Autre, à un audelà du récit inénarrable, à une relation intime entre les auteurs et les spectateurs, voire entre les spectateurs via les auteurs. Représenter ce «saut » est donc une gageure, cela consisterait à représenter l'irreprésentable: ce qui ne se peut qu'indirectement, en racontant au sein de la narration la contradiction, la misère et la vanité de la croyance en la justification finale des actes et par voie de réflexion, la vanité de la croyance en la narration. Le paradoxe étant que la mise en lumière de cette misère narrative par la narration est, pour reprendre les mots de Pascal, ce qui en fait la grandeur ${ }^{16}$. Pour ce faire, d'une part la série nous représente le "saut" comme tributaire d'une représentation passée d'un personnage via les flashbacks. D'autre part, comme dit précédemment, à chaque épisode, ce passé se redouble en se dissimulant à lui-même et en se comprenant sur le mode d'un présent itératif, habituel, semblable à un temps clos fait d'habitudes conservant un traumatisme par peur de s'ouvrir à l'avenir et accusant par làmême sa propre contradiction. La ferveur dans la série (et la ferveur en la série) devient ambiguë: est-elle confiance en le réel et en l'autre par-delà notre égoïsme ou déni dogmatique obsessionnel voire désespoir et ressentiment? 

propre relation au récit puisque raconter au sein de la narration la contradiction, la misère et la vanité de la narration et en appeler à un au-delà du récit (à sa fin), c'est concevoir celui-ci comme un sacrifice. C'est dans cette mesure que les auteurs nous interrogent sur le sacré et le saint, sur la frontière entre le sacrifice de l'autre pour soi (voire le sacrifice de soi contre l'autre), l'irreprésentable comme violence, et le sacrifice de soi pour l'autre, l'irreprésentable comme ouverture à l'Autre, via la référence biblique en général, et le chapitre $22 \mathrm{du}$ livre de $L a$ Genèse ${ }^{17}$ et les allusions à son exégèse kierkergaardienne qu'est Crainte et Tremblement ${ }^{18}$ en particulier. Lost nous offre-t-il un modèle de justification permettant de distinguer la croyance extravagante d'une croyance raisonnable, voire d'une révélation? Pour répondre à cette question nous nous focaliserons spécifiquement sur trois épisodes écrits par Cuse et Lindelof dans la deuxième saison. Premièrement, «Man of science, Man of faith » (S02E01) sera l'occasion de nous demander qui incarne cette opposition entre l'homme de science et l'homme de foi, et donc ce qu'elle désigne. Ensuite l'examen de «?» (S02E21) nous permettra d'examiner les résonances du désespoir de Locke avec le genre d'écriture auquel se réfère la série. Enfin, l'étude de l'épisode «Live together, Die alone » (S02E23-24) nous amènera à nous interroger sur la dimension éthique voire eschatologique de la croyance dans le choix du sacrifice de Desmond.

\title{
Man of science, Man of faith
}

\author{
Chez le réaliste, ce n'est pas la foi qui naît du \\ miracle, c'est le miracle qui naît de la foi. Si le \\ réaliste acquiert la foi, il lui faut, en vertu de son \\ réalisme, admettre aussi le miracle ${ }^{19}$.
}

Intitulé "Man of science, Man of faith", le premier épisode de la saison 2 oppose apparemment Locke et Jack quant à la valeur à accorder au bunker, opposition qui se « résoudra» - entre ces deux personnages - dans l'épisode «Orientation» (S02E03), quand Locke amène Jack à appuyer sur le bouton arguant de la difficulté du « saut de la foi ». Pour Jack, le bunker ne peut avoir qu'une fonction pragmatique et déterminée : abriter la communauté des survivants, les protégeant de l'hypothétique menace des Autres. Selon Locke, sa finalité est moins ponctuelle et locale que celle d'abri de fortune ; il est la raison d'être de leur présence sur l'île. Le titre du premier épisode nous incline à penser que John et Jack sont des archétypes, le religieux et le savant - couple qui n'est pas sans évoquer celui d'Ivan et Alexei dans le roman Les Frères Karamazov de Dostoïevski auquel il est fait explicitement référence dans l'épisode «Maternity Leave" (S02E15). Entre eux, sourd une dispute métaphysique : y a-t-il une finalité universelle à l'œuvre dans les séries d'événements et actions de chaque personnage, qui les harmoniserait ? Par voie de réflexion, ce problème a une résonance narratologique : y a-t-il une intelligence à l'œuvre ordonnant les épisodes, un narrateur providentiel prescrivant un sens unique au récit ou est-elle le produit d'un singe savant tapant sur une machine à écrire, d'un kaléidoscope combinant de façon aléatoire des narratèmes-clichés?

Pour Locke, le miraculé de la première saison, il existe une force directrice mystérieuse, une destinée, permettant d'interpréter une série d'événements en signes, épreuves et voies de salut. Dès qu'il rencontre Desmond, il croit au dispositif et se fait un devoir d'appuyer toutes les 108 minutes sur le bouton après avoir entré les numéros qui ont 
permis à Hugo (Jorge Garcia) d'être à la fois riche et misérable. Néanmoins, ses motifs sont ambigus: déjà durant la première saison, dans l'épisode "Deus ex machina » (S01E19), cette posture de croyance en une destinée est associée à la trahison de son père et à un ressentiment. Or dans l'épisode "Orientation» (S02E03), on découvre qu'en évoquant l'expression de "saut de la foi ", il répète une expérience passée : Helen (Katey Sagal), sa compagne, use de cette expression pour l'enjoindre à lui faire confiance en renonçant à prendre sa revanche sur son père.

17 Jack, le neurochirurgien, doute avant d'affirmer ou de rejeter un jugement. Il explique le cours des événements comme des faits en dégageant des lois générales sans se risquer à en examiner les causes premières et métaphysiques. Il ne croit pas au dispositif du Cygne et ne veut pas reprendre le rôle de Desmond assigné par la Dharma, estimant que son comportement est conditionné. Selon Sander Lee, Jack reproche à Locke sa mauvaise foi (dans le sens de l'existentialisme sartrien), l'accusant de se déresponsabiliser, de renier sa liberté subjective, de choisir de renoncer à choisir ${ }^{20}$. Toutefois, Jack est aussi de mauvaise foi en prétendant choisir en fonction d'une liberté métaphysique égoïste et solitaire informant un monde indifférent qui n'a de sens que pour soi, car il renie alors son désir d'Autrui, refusant de reconnaître la part qu'occupe ce dernier dans ses propres décisions. S'il ne croit pas dans le bunker, c'est parce qu'il reconnaît l'individu Desmond : il s'est fié à lui par le passé, dans un sens différent et plus intime, que celui d'une croyance héroïque dans le salut du monde... et il ne veut pas réitérer cette expérience.

Objectivement, que l'action d'«entrer les numéros" ait pour résultat de "sauver le monde» peut paraître improbable. Au mieux sauve-t-on une partie de l'île et les survivants. L'observation du dispositif - un décor désuet, vestige des années 1970, un ordinateur dépassé dans son dôme, un film d'orientation $3 / 6$ en super 8 ressemblant à un film d'entreprise New Age - et de l'accaparement excité de Desmond peut mener à la conclusion d'un conditionnement. Il s'est persuadé qu'il sauvait le monde à force de répéter la même séquence sensori-motrice. Ayant contracté une habitude, c'est-à-dire une aisance à la réalisation de celle-ci, la justification qu'il lui prête pour la perpétuer est idéologique. Le rite précède le mythe, pourrait-on dire.

L'hypothèse est d'autant plus vraisemblable que l'ouverture de la saison nous montre un individu sans visage, un corps surentraîné pris dans un quotidien mécanique et réagissant à son milieu dans une activité infatigable et sans but. D'autre part, l'œil qui ouvre chaque saison de la série n'est pas le premier élément signifiant de l'épisode mais le second: l'ouverture de l'œil est la réaction motrice de Desmond à un stimulus sonore originellement invisible, le signal sonore du compteur. Sarah Hatchuel met en lumière, dans son analyse de ladite séquence, que la continuité de la bande son de The Mamas and the Papas - ni tout à fait in ni tout à fait off - nous présente un temps de l'itération contrastant apparemment avec les paroles mêmes de la chanson, ode à l'originalité du comportement ${ }^{21}$. Cette opposition audiovisuelle entre l'uniformité anonyme et la revendication d'une singularité exceptionnelle n'est pas tant une contradiction logique qu'un paradoxe : plus il se croit spécial, plus il se répète, fait la même chose, et vice versa. C'est la logique délirante du bunker dont cette séquence nous donne la clé et qui n'est pas sans évoquer la logique souterraine de la haine et de l'auto-déception animant le narrateur bipolaire et paranoïaque des Carnets $d u$ sous-sol de Dostoïevski.

Cependant les flashbacks de l'épisode « Man of science, Man of faith » nous montrent que Jack refuse de croire au bunker, car un événement incroyable lui est arrivé. Lui-même a été déçu par une promesse et surtout s'est lui-même déçu. Comme le déclare Lindelof 
dans les bonus DVD de la saison 2, l'opposition homme de science/homme de foi ne désigne pas tant Jack et John que les deux faces d'un même Jack. Le médecin doute du dispositif du Cygne non pas tant par exigence rationnelle - il fait l'expérience de l'attraction magnétique de la clé de la mallette d'armes contre le foyer de l'incident mais parce qu'il a été témoin d'un miracle, la guérison de Sarah (Julie Bowen). Néanmoins ce miracle n'est pas la conséquence d'une orthodoxie, de la croyance en une destinée objective. Il repose sur une promesse faite à Sarah relevant d'une situation éthique limite. Celle-ci est analogue à celle de la confiance de Locke en Helen puisque dans cet épisode Jack renonce ponctuellement à sa rivalité avec son père et s'efforce de comprendre le sens de son conseil. C'est parce qu'il a cru à un miracle salvateur qu'à présent Jack doute du salut par le bunker. Son doute est tout à la fois un état de plus grande lucidité et d'aveuglement sur son rôle dans ce miracle passé. Jack est plus lucide car il a compris que l'événement extraordinaire ne dépendait pas de lui. Toutefois ce réalisme prétendant faire le deuil de son omnipotence dissimule un état de désespoir par lequel il s'aveugle. En effet, ledit événement n'a pas dépendu de lui ; Jack le regrette et conserve ce regret comme une revanche. Ainsi se complait-il dans l'assurance de son impuissance, se vengeant de la sorte contre l'espoir de l'autre (Christian, Locke, Sawyer), voire contre l'espoir que l'autre (Sarah, Kate) fut pour lui, en décrétant impossible tout miracle.

21 Aussi le dualisme statique " homme de science et homme de foi » ne tient-il pas. La série rappelle que la frontière entre savoir et croire n'est pas si hermétique, qu'il s'agit plutôt des attitudes corollaires interrogeant le rôle du doute. C'est pour cela que le personnage qui résout la crise, Desmond, est l'homonyme d'un philosophe écossais se qualifiant de sceptique modéré, David Hume. En effet, on peut définir la croyance comme une attitude propositionnelle qui nous fait affirmer un jugement comme valable pour nous, donc sous conditions, plus qu'en soi, absolument. Elle dépend d'une disposition subjective sur la base de laquelle on assent ou nie une proposition possible. Par cet acte, on suppose l'existence ou l'inexistence pour nous du contenu de ladite proposition. Or, par opposition, le savoir, selon la fameuse définition platonicienne du Théetète, est une croyance vraie (objectivement, réellement) et justifiée, accompagnée de sa définition ${ }^{22}$. Aussi permet-il de penser l'existence indépendante d'une réalité partageable constituée d'entités à l'identité stable et durable.

Croyance et savoir peuvent être alors considérées comme deux attitudes subjectives par rapport à la manière d'affirmer ou de nier des jugements. Dans la croyance pure, la validation du jugement dépend surtout de l'assentiment subjectif particulier : celui qui affirme ou nie le jugement peut ne pas savoir s'il est objectivement vrai ou faux et ne pas le démontrer, c'est-à-dire ne pas exhiber auparavant les causes objectives suffisantes dudit consentement. En revanche, dans le savoir, l'assentiment subjectif particulier au jugement passe par la médiation de l'universel, c'est-à-dire qu'il succède à la mise en évidence de sa vérité, et non de sa fausseté, ainsi que de conditions objectives suffisantes : d'une définition partageable préalable des objets du jugement et des propriétés qui leurs sont prédiquées, de la non contradiction avec des jugements déjà connus et de la possibilité de le ramener à un état de fait observable. En termes cartésiens, celui qui sait retient sa volonté d'accepter ou de nier une représentation en la soumettant à son entendement : il doute donc, tandis que celui qui croit fait passer sa volonté avant toute explication objective. empiriques car liées à notre sensation et notre imagination que le savoir peut certifier, 
voire qu'il peut corriger comme étant une erreur. Et ce, selon un principe de réalité, c'està-dire en limitant notre tendance égocentrique à voir le monde à la mesure de notre désir particulier, en rappelant l'existence objective et indépendante d'un réel hors de nous qui serait le même pour tous ou en soi, indifféremment. Dans cette optique, Jack a un comportement « cartésien » dans le flashback qui lui est consacré au premier épisode de la saison 2 : il rend son diagnostic à Sarah en se soumettant à une nécessité statistique et objective. Il annonce les issues probables de l'opération qu'il va réaliser avec le détachement et l'expertise d'un technicien. Cela lui sera reproché par son père, Christian (John Terry), comme une réserve inappropriée car il ne s'adresse pas à un objet mais à un autre sujet : dans cette situation, la vérité et la justification de l'énoncé ne dépendent pas simplement de l'exactitude informationnelle. L'annonce doit prendre en compte l'attente de l'interlocuteur, comme le ferait le narrateur d'un récit avec son audience. Or Jack entend d'abord ce conseil comme celui d'un recours à de la rhétorique quand il rabroue son père car l'attente que va susciter la promesse de guérir Sarah est démesurée d'un point de vue quantitatif, même si qualitativement c'est cela qu'elle veut entendre. Il a raison: c'est de la rhétorique, mais elle est paradoxale. D'une part, elle implique la conscience de l'éminente improbabilité de l'issue, voire de son impossibilité. D'autre part, elle ne prend en compte que l'attente du destinataire en tant que telle, l'attente en tant que pure ouverture au possible d'un être unique et non en tant que prédiction de quelque chose pour quelque intérêt particulier. La conscience aiguë de la perte en vue de la pure attente du destinataire distingue également cette promesse d'un engagement éthique normal, du point d'honneur, présupposant qu'à l'impossible nul n'est tenu. Ici il ne dépend pas que de lui que ce qui est promis, soit tenu, mais de la confiance en celui à qui l'on fait la promesse. Cela ne fait donc pas seulement courir le risque à Jack de ne pas être à la hauteur de l'excellence et de l'intransigeance fantasmées de son père, mais d'une perte telle qu'elle dévalorise cette dernière obsession. Il y aurait donc un autre type de croyances que le discours rationnel ne pourrait totalement résorber. Celui-ci repose sur des principes et des définitions d'objets absolus que l'on doit accepter pour démontrer le reste mais dont on ne peut prouver l'existence ni l'inexistence sans paralogisme.

"Croire à sa perte est impossible. Comprendre qu'humainement c'est sa perte et en même temps croire au possible, c'est croire ${ }^{23}$. » Cet autre type de croyances serait de l'ordre de la foi, c'est-à-dire d'une conviction intime catégorique et singulière qui s'apparente à ce que Kierkegaard nomme "saut " pas seulement dans l'inconnu, l'imprévu, mais dans l'absurde ${ }^{24}$. L'absurde n'est pas simplement le mode objectif de l'impossible logique du type « le cercle carré ». C'est au contraire du pur possible, mais qui excède les conditions de la démonstration objective. Il est littéralement improbable, quoique nécessaire à toute démarche de justification rationnelle. L'absurde est donc un paradoxe spéculatif ouvrant à l'attitude éthique de la résignation, voire à une interrogation métaphysique qui s'enracine, ultimement, dans l'expérience intime de la finitude, de l'isolement, de la perte de soi. Cette expérience est ce que Kierkegaard nomme la "tonalité affective» de l'angoisse ou du désespoir - qu'on pourrait exprimer poétiquement par le fameux « To be or not to be » du Hamlet de Shakespeare. Cette disposition se ressent comme entrevision de l'absolu, de l'éternité, à travers la relativité des apparences et leur devenir. L'éternité, en sa transcendance, sa différence d'avec le monde des phénomènes, est entraperçue comme éminente possibilité du néant dudit monde. Plus prosaïquement, tout ce qui se déploie dans le temps devient, pour l'angoissé, contingent, susceptible de n'être qu'une illusion sans rime ni raison, voile de Maya, sans densité ontologique. 
D'un point de vue éthique, cette possibilité pure se résout en nécessité, impératif catégorique. L'absurde se comprend alors sur le mode de la résignation et de la «dure réalité » qui est celle du devoir moral voire civil (grosso modo le rigorisme kantien) : la volonté individuelle doit se faire une raison. Il faut renoncer à expliquer certains problèmes théoriques, comme il faut renoncer à faire dériver les obligations morales de maximes de l'intérêt particulier. L'autonomie consiste à se soumettre à une pure volonté abstraite, le devoir, en faisant taire ses sentiments naturels et la question du bonheur. Ainsi Agamemnon se résigna-t-il à sacrifier Iphigénie car c'était nécessaire à la communauté. Autonome, il fit passer l'intérêt des Grecs avant son propre intérêt, c'était sa vertu. Le Tout prime sur la partie, il faut obéir pour obéir. Mais cette résignation est désespérante, et cette condition, celle de l'homme moderne, est parfaitement illustrée dans la série par la figure de l'homme du bunker. Celui-ci est une allusion à «l'homme du souterrain», petit fonctionnaire de Saint-Pétersbourg, narrateur mégalomane et impuissant, rongé par l'envie et la haine de sa résignation des Carnet du Sous-Sol de Dostoïevski ${ }^{25}$.

Le « saut de la foi » consiste à se résigner à ne pas se résigner et vouloir l'Être en prenant pleine conscience de son improbabilité, c'est-à-dire en pleine conscience de la perte. Selon l'exégèse problématique de Genèse 22 que produit le philosophe danois dans Crainte et Tremblement, seul l'individu qui s'est résigné au général et qui simultanément et malgré tout affirme l'individuel, est fervent, sachant qu'il affronte alors l'angoisse en se confrontant immédiatement à l'absolu ${ }^{26}$. La récurrence des thèmes et des termes d' « île " et d'« Autres » montre que la série présente clairement une métaphysique où la question de la transcendance de l'Être est déterminée par celle de l'Autre. "Où est ton frère?" telle est la question biblique de Genèse 4 qui tourmente Jacob et l'origine du « Tu ne tueras point » empêchant l'homme en noir d'éliminer les candidats (et qu'il s'efforce de contourner en permanence); question au fondement de la métaphysique, selon Lévinas, précédant la question ontologique angoissante « Pourquoi y a-t-il quelque chose et non pas rien? ». L'angoisse est angoisse de la mort d'autrui ${ }^{27}$. Dans ce cadre, la promesse de Jack montre le changement d'attitude que le personnage prend avec autrui, découvrant que c'est la relation qui fonde son identité propre. C'est également à une telle démarche que fait manifestement référence Helen à Locke, en le priant de renoncer à en remontrer à son père, puis Locke à Jack, en l'implorant d'appuyer sur le bouton.

Dans « Man of science, Man of faith », Jack est homme de foi car, pour la première fois, il nourrit un souci d'autrui qui ne serait pas lié à son amour propre. Les allusions du second flashback au chapitre 18 du livre de la Genèse témoignent qu'à ce moment il n'a plus affaire à un exemplaire particulier du type « patient » qu'il doit réparer mais bien à autrui en sa transcendance: Sarah se révèle en cet instant de promesse, par son rire, comme étant la patience même. En effet, après son entretien avec Kevin (Anson Mount), le fiancé de Sarah, qui annonce qu'il la quittera si elle n'est plus sexuellement « opérationnelle » et donc apte à avoir des enfants à la suite de l'intervention, le médecin prend le risque de promettre l'improbable, sa guérison, et engage sa responsabilité auprès d'elle car il entraperçoit son attente. L'attente a ici un sens indissociablement moral (Jack entraperçoit sa responsabilité dans le bien être et la dignité de Sarah), et physique (l'attente ou «expect» en anglais désignant ici la grossesse). C'est pourquoi la promesse du médecin la fait rire car elle-même alors ne se résigne plus à la résignation à laquelle leur premier entretien l'avait conduite. Or c'est dans un moment d'incertitude quant à l'issue de ladite promesse qu'il rencontre Desmond en se reprochant d'avoir promis tout 
en sachant que ce qui était promis était quasi impossible car il est certain d'avoir effectivement échoué. Par une certaine logique de l'absurde, du "pourquoi pas?», en lui demandant ce qu'il en serait s'il avait réussi malgré tout, Desmond ne nous semble pas simplement lui rappeler que tout est relatif, mais surtout relationnel. Ainsi une promesse ne se comprend-elle pas uniquement par le constat d'un état de fait objectif mais par l'interprétation qu'on est susceptible d'en donner pour l'autre, voire qu'est susceptible d'en donner l'autre pour nous.

Le miracle, « objet » de foi, relève alors d'un impératif moral limite nous faisant basculer dans l'ordre du métaphysique. L'avènement de ce qui est promis à l'autre et pour l'autre suppose le sacrifice de sa philautie par celui qui promet tout autant qu'un événement réel admirable. Cependant cet événement est merveilleux car unique et transcendant, c'est un avènement, une épiphanie. De fait, on ne saurait le répéter sans risque, c'est-à-dire sans que le personnage ne l'attribue à ses propres capacités et sans que la relation à l'autre ne tombe dans le rapport. C'est ce que comprennent Jack et John dans les flashbacks qui leur sont consacrés, dans les épisodes "The Hunting party» (S02E11) et «Lockdown" (S02E17) : ceux-ci perdent les femmes qu'ils aiment en voulant répéter et contrefaire cette prise de risque qui les avait unis et ne cesseront, dans tous les autres flashbacks de la série, d'être les agents de leur chute. Ils veulent répéter cette première fois car ils refusent d'être aimés en retour et, reprenant l'alibi de la rivalité avec leur père, préfèrent rétablir un rapport bien unilatéral entre eux et l'aimée plutôt que d'assumer la réciprocité d'une relation. Ils sacrifient ainsi l'aimée pour laquelle ils s'étaient d'abord sacrifiés et embaument le « saut » de cette première fois, de cette promesse, pour n'y plus voir qu'un « incident ». Et c'est autour du foyer de cet « incident », de la promesse comme perdue, que s'échafaude un sarcophage de hiéroglyphes. Ce dernier est l'ensemble des discours d'auto-justification des comportements envieux des personnages, c'est-à-dire l'ensemble de leurs flashbacks. Par ceux-ci, les personnages prétendent congédier la perte sur le mode de la répétition, plutôt que de reconnaître qu'eux-mêmes ont fait de cette promesse une perte.

De même, au niveau de la réception et de l'énonciation de la fiction, en répétant ce miracle, le risque est que les spectateurs curieux l'attribuent au savant calcul d'un auteur qui pourrait alors s'autoriser à être ce démiurge. Dans cette illusion rétrospective, il nous semble que le résultat inattendu de la loterie est déjà contenu dans le tirage et le choix des numéros; nous oblitérons alors l'incertitude vécue qui l'aura rendu possible pour reprendre une comparaison de Kierkegaard également exploitée dans la série ${ }^{28}$. Si le penseur danois parle de "saut ", c'est d'ailleurs qu'il ne peut pas simplement définir la foi conceptuellement (puisque l'on excède le cadre de la démonstration), et qu'il prend acte $\mathrm{du}$ fait qu'il faut cependant communiquer et répéter cette expérience d'où une écriture autobiographique aux airs d'homélie et de confession, faite de commentaires de fictions, de faits divers d'écrits bibliques et d'expériences personnelles ${ }^{29}$ - ce qui interroge, pour la série, la faculté du récit à rapporter ces miracles, ces rencontres intimes, pour les faire revivre et partager au public. Or cette reprise est ambivalente, car elle peut faire de ses personnages ou de son auteur des thaumaturges, et des spectateurs, une secte dogmatique. Ce qui, toujours selon le philosophe danois, est le problème de la répétition, rituel que la série illustre avec la réactivation du compteur toutes les 108 minutes dans le sarcophage contenant l'incident ${ }^{30}$. miracles quoiqu'ils soient tentés de se prendre pour des héros solitaires. Cela se retrouve 
dans la séquence de la rencontre entre Jack et Desmond qui se clôt sur un type de jeu de mots récurrent dans cette saison. L'Écossais, en soignant la cheville de Jack, lui conseille : "You have to lift it up », soit « Tu dois la lever (ta cheville) », mais il a un tel accent que l'on entend, avec son interlocuteur : "You have to lift her up », soit : « Tu dois la lever (Sarah)». Ce trait d'humour révèle à Jack que son dévouement pour Sarah n'est ni le fait d'une parole d'honneur toute formelle s'opposant à une pulsion animale, ni d'une pulsion animale aveugle se parant des oripeaux de valeurs chevaleresques, mais d'un amour qui ne doit pas être stérile. Il n'est pas un héros épique, ni tragique quoiqu'il en semble. Il n'est pas une belle âme qui répare tout mais avec qui tout le monde est injuste (Sawyer, Kate, Sarah et surtout son père) : ça c'est le récit flatteur que la série s'attache à fêler ${ }^{31}$. Toutefois il faut qu'il promette à Sarah et qu'il appuie sur le bouton pour répéter cette promesse et commencer à le comprendre. Cela signifie également que, pour le discours imaginaire qu'est le récit de la série, foi singulière, savoir général et croyance particulière sont peu discernables sans l'engagement du spectateur, tout en sachant qu'il n'épuisera pas le sens du récit, ce que souligne Pacôme Thiellement pour qui le problème de Lost repose sur l'opposition confiance/défiance voire la dialectique, l'oscillation, entre ces deux sentiments dont l'opposition croyance/connaissance est la forme de justification secondaire $^{32}$. En effet, l'un des thèmes majeurs du récit est la perte, perte non seulement physique, mais morale voire métaphysique, le ressentiment, le désespoir comme mal contemporain $^{33}$.

Toutes les actions humaines seront d'elles-mêmes classées, selon ces lois, mathématiquement, un peu comme des tables de logarithme jusqu'à 108 000, elles seront inscrites à l'almanach ; ou, mieux encore, on pourra voir paraître des éditions utiles du genre de nos dictionnaires encyclopédiques, où tout sera noté et codifié avec une telle exactitude qu'il n'y aura plus jamais d'actes, ni d'aventures ${ }^{34}$.

La suspension volontaire d'incrédulité est particulièrement critiquée dans l'épisode 21 intitulé «?», dans lequel Locke et Eko (Adewale Akinnuoye-Agbaje) découvrent une troisième station qui offre une réflexion sur l'énonciation et la réception de la narration non seulement de la première et deuxième saison mais également de toutes celles qui suivront car la crise ouverte ne sera jamais totalement résolue, sinon par une assertion sibylline de Hume : «But you saved my life, brother, so that I could save yours ${ }^{35}$.». La station se trouve sous un avion biplan contenant des statuettes de la Vierge remplis de cocaïne, ambivalence ironique de l'objet de croyance religieuse, présenté à la fois comme signe de mystère (le point d'interrogation) et comme drogue, opium du peuple, ne servant qu'à le divertir de son désespoir ${ }^{36}$. Le film d'orientation en Betacam annonce que les membres de la station du Cygne croient se livrer à une tâche très importante et qu'ils font l'objet d'un test psychologique sur la soumission à l'autorité ; il s'agira pour les membres de la station de La Perle d'observer et de noter leurs faits et gestes. À cette révélation, Locke considère que Le Cygne n'est qu'une illusion, un simulacre se jouant pour des regards sadiques voire des fauteuils vides et que les hommes du bunker ne sont que des rats de laboratoire. Désemparé, il considère qu'il est victime de sa propension à imaginer le monde comme 
conforme à ses désirs et, a contrario, le réel lui apparaît comme néant, un show creux, un jeu d'effets contraires sans rime ni raison. Sa rage est d'autant moins contenue qu'Eko, le vrai faux prêtre catholique, répète l'enthousiasme que Locke lui-même professait auparavant et quasiment pour les mêmes raisons - raisons que sa haine lui montre à présent comme étant débiles. C'est sur la base d'un rêve (justification qu'admet pourtant Locke dans la série) que le nigérien se dirige vers la station et accepte d'appuyer sur le bouton. Il est manifestement un double de Locke et donc son rival en termes de ferveur et de vocation sacrée. Dès lors, Locke, à qui nul ne doit dire ce qu'il ne peut pas faire, veut se distinguer de ce double à tout prix, quitte à se détruire, quitte à tout détruire.

En effet, Eko infère des impressions de l'entrée continue de la série de numéros et du film d'orientation, à l'importance de la tâche en question. Si l'activité est contrôlée, si elle se donne à voir, alors sa répétition est importante pour celui qui regarde et qui est susceptible d'y trouver un sens, contrairement à celui qui la répète, qui peut douter dudit sens de ses actions. D'autre part, en tout état de cause, l'énoncé du film d'orientation est tel qu'il nous apprend que ce qui est l'objet du test psychologique est la croyance des participants en la valeur de l'opération d'entrer les numéros. Or cela ne signifie pas nécessairement que l'objet de ladite croyance soit faux.

D'une manière générale, le type d'énonciation du récit de Lost favorise cette mise en crise du principe de réalité et du rapport à la connaissance. En effet, la série multiplie les focalisations internes sur des événements propres censés se réintégrer dans une trame globale, voire convoque des points de vue contraires sur un même ensemble d'événements, de telle sorte que celui-ci peut changer de valeur. Cette confrontation de points de vue dissonants nous amène à nous représenter la coexistence comme une crise de la perception du réel pour chacun. D'ailleurs, si l'on se reporte aux analyses de Todorov, la série suit plutôt les codes de composition du genre fantastique mâtiné de science-fiction $^{37}$. Son écriture imite le ton documentaire, de l'archive ou du journal retrouvé - la démarche archéologique où l'on explore les vestiges des stations et visionne des vieux films d'orientation Dharma, pour présenter un versant factuel voire réaliste objectif, tout en adoptant la perspective d'un personnage dans la fiction, ce qui relativise l'objectivité et permet de faire perdurer les hésitations des spectateurs. Ces derniers ne savent alors pas s'ils doivent attribuer aux événements racontés une cause surnaturelle, une cause psychologique imaginaire ou réelle mais encore inexpliquée vus les moyens techniques et théoriques dont nous disposons à présent, jusqu'au moment du dénouement et encore...

Une telle ambiguïté se retrouve particulièrement dans un classique du fantastique auquel cette saison ne cesse de faire référence, Le Tour d'écrou (1898) de Henry James. L'ouvrage dissimule d'ailleurs le film d'orientation de la station du Cygne. Dans cette nouvelle, l'auteur joue sur l'incertitude quant à la nature des événements racontés à travers une multiplication de voix assurant le récit et une distance temporelle entre les deux instances narratrices déléguées, ainsi qu'à travers une réflexion (par l'une de ces voix) sur un déterminisme psychosexuel présidant à l'énonciation de la seconde. Ainsi, la narratrice déléguée raconte l'histoire de fantômes qu'une autre, une gouvernante, lui a raconté il y a plusieurs années. Mais simultanément, elle soulève l'hypothèse d'une hystérie hallucinatoire dont souffrirait cette « vieille fille ». De là, le titre anglais Turn of the Screw, porteur d'un double-entendre, pourrait aussi se traduire par Tour de baise. Ce jeu de mots supposerait que la nouvelle pourrait être comprise comme un exercice d'écriture formel, une configuration d'effets totalement creux et indifférents à son énoncé et visant 
à défier les lecteurs qui seraient tentés d'identifier ce qui serait réel ou $\mathrm{pas}^{38}$. Dans sa deuxième saison, la série pousse cette logique réaliste de la distance temporelle et de l'explication déterministe du vécu des personnages jusqu'à un point critique menaçant la réalité même du raconté. Effectivement l'explication déterministe elle-même se mue en possible entreprise de manipulation. La narration dissout d'une main la croyance première du récepteur tout en l'exigeant de l'autre. L'ambiguïté fantastique devient alors ambivalence d'un réalisme paranoïaque : on n'hésite plus sur la nature d'un événement pour un personnage mais du réel lui-même pour plusieurs personnages qui eux-mêmes sont discordants les uns avec les autres et avec eux-mêmes.

Le recours aux principes de la cybernétique appliqués à la sociologie et à la psychologie évoque, par sa prétention même à l'objectivité clinique, une entreprise de déshumanisation aux relents concentrationnaires. La reprise des configurations audiovisuelles éculées du documentaire pédagogique offre un regard si abstrait qu'il nous fait basculer du fantastique dans un univers où le réel objectif s'opposant à la croyance subjective particulière ne serait qu'une construction sociale à visée normative, une idéologie. Mais cette mise en crise du réel est le corrélat de la désintégration de l'identité des personnages qu'on découvre de plus en plus divisés sans qu'eux-mêmes ne daignent l'admettre ; les flashbacks et autres configurations temporelles nous montrent qu'ils font toujours plus la même chose. Là encore, le monde et les narrateurs délégués sont comme le maniaco-dépressif qu'est le narrateur des Carnets du Sous-Sol pour qui l'empire de la techno-science et la morale utilitariste du monde moderne sont les ressorts d'une oppression qu'il hait car portant atteinte à son éminente singularité, singularité qui se délite pourtant dans son acrimonie impuissante. La reprise des codes de représentation des expérimentations de l'éthologie behavioriste - comme les cages de Skinner par lesquelles il conditionnait ses rats - et de l'expérience psychosociologique de Stanley Milgram sur la soumission à l'autorité et la spirale d'engagement - à laquelle fait manifestement référence la station Perle - font de notre croyance en le monde raconté via les personnages (et, par effet de réflexion, de notre croyance dans le monde réel), le résultat d'un conditionnement réifiant, malveillant, totalitaire.

Ces configurations nous dévoilant l'oppression normative sous le réel suppose une narration qui se focalise sur des personnages de plus en plus en lutte contre eux-mêmes. La plupart des flashbacks et autres variations temporelles du récit (à l'exception sans doute de ceux de Jin et Sun et de Bernard et Rose) présentent des événements traumatisants, quand l'impossible devient possible, pour reprendre la terminologie de Kierkegaard. Or les promesses que portaient les événements heureux étant souvent préludes à une perte provoquée par le personnage lui-même, ce dernier tendant à répéter cet échec sur l'île. D'ailleurs par la ressemblance entre les flashbacks de la première saison et ceux de la deuxième, le récit nous montre des protagonistes qui se caricaturent eux-mêmes et s'enfoncent davantage dans une persona unidimensionnelle (Michael et Sawyer en tête sans doute) devenant, d'eux-mêmes, les rats de laboratoire qu'ils prétendent ne pas être. Leur comportement est obsessionnel car ils se mettent dans des situations de double contrainte : ils veulent réitérer le passé comme passé, jouant de la sorte à "qui gagne perd». Pour que leur action "réussissent», ils se placent dans un contexte analogue à celui dans lequel leur action passée avait échouée. Dans les termes de l'écologie de l'esprit de Bateson, ils retrouvent des situations perdant-perdant en se mettant en demeure d'accomplir une injonction qui suppose une confusion de types logiques et ne peut être réalisé qu'en y dérogeant ${ }^{39}$. Ce contexte de violence 
psychologique schizogène par communication paradoxale est d'ailleurs un principe de l'écriture de Joseph Heller dans "Catch-22", roman que trouve Desmond en sauvant Naomi (Marsha Thomason) dans l'épisode justement intitulé « Catch-22 » (S03E17).

La double contrainte s'inscrit dans une situation de communication où la confusion des types logiques d'un message et des contextes d'énonciation est inévitable et délétère pour l'interlocuteur, entraînant son autopunition systématique. Il faut deux personnes ou plus; une expérience répétée; une injonction négative primaire; une injonction la contredisant plus abstraitement, mais tout aussi négative; une injonction tertiaire interdisant à l'interlocuteur de quitter la situation. Bateson remarque que le schizophrène et l'artiste ont en commun une tendance à confondre les types logiques (ce qui est le ressort des figures poétiques) mais que cette confusion est positive, belle et morale, car souple, chez l'artiste. Comme on l'a vu avec le jeu de mots de Desmond, «Lift her up ", l'un des enjeux de Lost est de révéler la violence de ces contradictions pour les désamorcer et assouplir ces situations dites perdant-perdant par l'humour et par le récit et ses virtualités métaphoriques, révélant ce fond de violence et susciter de nouveaux types de réponses.

En ce sens la série propose une relecture postmoderne du trouble du principe de réalité de l'univers rationnel moderne dans l'art baroque : dans l'épisode « 316 »(S05E06), Ben, le manipulateur, fait l'éloge du saut de la foi. Il commente l'énoncé du chapitre 20 de l'Évangile de Jean: " Parce que tu m'as vu, tu as cru; bienheureux ceux qui, sans avoir vu, ont cru. » en prenant paradoxalement l'exemple d'un tableau, L'Incrédulité de Saint Thomas (1604) du Caravage, pour révéler qu'il ne faut pas voir pour croire ${ }^{40}$. Ce faisant, la série met en exergue la démarche artistique voire iconographique hautement ironique du maître italien dont Poussin disait que l'œuvre relevait d'un effort pour détruire la peinture. Néanmoins, c'est surtout au théâtre européen de la fin du XVI ${ }^{e}$ et du début du $\mathrm{XVII}{ }^{\mathrm{e}}$ siècle auquel Lost fait référence. La série reprend à nouveaux frais le thème du theatrum mundi de Calderón, du théâtre tragique et des romances de Shakespeare et des comédies fantaisistes de Corneille. Le théâtre représentait alors le monde dans une boîte entre symbolisme et illusion, comme un ensemble d'images mnémoniques, de chiffres (ou stations) nous donnant accès à une culture occulte mais aussi comme un jeu mécanique d'apparences sans fond objectif, un ensemble de phénomènes évanescents et vains n'existant que pour le regard des spectateurs. Ainsi, la thématique récurrente de ces œuvres est l'interrogation sur l'existence même d'une trame voire d'un auteur qui la filerait, c'est-à-dire d'un individu faisant autorité : un roi, un mage que l'on tend à confondre avec un usurpateur machiavélique et un illusionniste, un deus absconditus comme le Prospéro sur son île dans La Tempête, que l'on tend à confondre avec un Malin Génie, son frère Antonio. Ce thème est si efficacement traité que régulièrement fleurissent des critiques historiques et littéraires spéculant que Molière ou Shakespeare n'étaient que des usurpateurs voire les prête-noms d'auteurs connus de quelques happy few.

39 La référence au théâtre et à l'autorité du poète anglais est toutefois manifeste ${ }^{41}:$ d'une part, The Swan (le Cygne) est le théâtre dans laquelle Shakespeare présenta ses premiers drames avant le Globe - volume géométrique dont la forme pourrait évoquer la forme d'une perle et de l'œil qui regarde -, d'autre part, la forme circulaire du logo de la station La Perle renvoie à celle de la scène élisabéthaine. Les stations fonctionnent donc de concert : l'une serait la synecdoque de la série et l'autre celle qui met en abîme le rôle du spectateur nécessairement tiraillé entre l'incrédulité devant une supercherie, une 
trahison, et une volonté de croire malgré tout au récit et en un auteur. Ce tiraillement atteint son apogée à la fin de la saison 2, dans l'épisode "Live together, Die alone », où la question est de savoir si appuyer sur le bouton est une illusion dans la mesure où tout est filmé (donc construit pour un regard), une observation objectivant les actes, émotions et perceptions des personnages en une collection de données et réduisant le comportement à une série d'automatismes dénués de finalité. Par voie de réflexion, l'univers de la série consisterait en un agrégat de narratèmes, de promesses disparates valant comme autant de ballons d'essais, combinés de façon aléatoire pour susciter l'activité quasi obsessionnelle de la part du spectateur, dérivant d'une répétition de signaux et n'ayant d'unité synthétique que par la seule projection de notre désir. Comme l'a écrit Pacôme Thiellement, dans la station La Perle, c'est effectivement le regard du spectateur qui est interrogét ${ }^{42}$. Il est appelé à travailler à se rendre voyant, selon la terminologie de Jean-Luc Marion dans L'Aveugle à Siloé ${ }^{43}$ : c'est-à-dire qu'il est appelé à se découvrir à lui-même sa position de voyeur supposant un rapport unilatéral du Moi à l'Autre. Sans cela il oscillera sans cesse entre la Perle et le Cygne dans son rapport aux auteurs. Soit il croit que son œil seul fait le spectacle comme l'huitre crée la perle à partir des grains de sables épars auxquels se ramène un récit qu'il aurait pu écrire et comprendre mieux que ses auteurs. Soit il croit que le spectacle existe tout à fait sans lui, créé par un divin auteur semblable au cygne serein qui passe et reflète la lumière pour reprendre les mots de Proust dans Jean Santeuil, c'est-à-dire un auteur qui en sait plus que lui sur le récit et qui bien sûr en sait plus sur lui, le spectateur ${ }^{44}$. Dans les deux cas, c'est le même refus de considérer que l'œuvre prend sa valeur dans la relation de l'auteur et du spectateur, dans la proximité de l'Autre, en se persuadant plutôt que la création du récit vient d'une de ces deux instances à l'exclusion de l'autre.

40 Ainsi grande est la tentation de confondre La Perle avec l'œil totalitaire, celui qu'Anna Lucia découvre dans la Station de la Flèche dans « The Other 48 days » (S02E07) et dont on apprendra dans la saison 3 qu'il appartient à Mikhail Bakounine (Andrew Divoff), homonyme du révolutionnaire russe. Cet œil de Moscou voit la société comme le «palais de cristal » de Tchernychevski qui excitait tant la paranoïa du narrateur des Carnets $d u$ Sous-Sol de Dostoïevski. Pacôme Thiellement reproche ainsi aux détracteurs de Lost, et surtout aux inquisiteurs zélés devenus apostats au dernier épisode de la saison 6, cette asymétrie de leur regard sur la série : ils désiraient lire le récit sans se laisser lire euxmêmes par le récit. Être lu signifie ici reprendre et faire travailler les symboles par-delà les illusions d'omnipotence qu'ils pourraient procurer quand on essaye d'y trouver un sens absolu et exclusif caché mais encodé car accessible aux seuls initiés, aux happy few de l'initiative. Il est donc nécessaire de renoncer au contrôle solitaire - le « let go » par lequel tous les personnages, à commencer par son père, serinent Jack au cours de la série ${ }^{45}$ pour nourrir une confiance bienveillante en l'Autre.

41 En présentant ce jeu de double contrainte entre la Perle et le Cygne, entre un regard objectif désenchanté et oppressant et un regard fervent, entre Antonio et Prospero, John et Jack, la série prétend aussi à un dépassement. Cuse et Lindelof n'ont pas oublié que, dans La Tempête, entre Prospero et Antonio, il y a Miranda («Admirant » en italien) qui réconcilie les frères ennemis en tombant amoureuse de Ferdinand. Ainsi, entre Locke et Jack, il y a Desmond et Pénélope (Penny) (Sonya Walger). Il s'agit donc bien de retrouver cette confiance naïve dans le monde, renoncer au contrôle, à la vengeance ou à la rétribution : l'attitude de Desmond, dans les deux derniers épisodes de la saison, indique la voie. 


\section{Live together, Die alone}

«Mourir pour», « mourir pour l'autre ». J'ai pensé
aussi, à un certain moment, à appeler mon propos
" mourir ensemble ${ }^{46}$ ".

Au début de la première partie du double épisode final de la saison 2, nous apprenons le nom complet du mystérieux Desmond qui est Desmond David Hume, homonyme du philosophe écossais empiriste du XVIII ${ }^{\mathrm{e}}$ siècle qui se définit lui-même comme sceptique modéré. Pour Hume, nos connaissances de faits reposent sur des relations d'idées comme la causalité et l'identité dont on ne peut démontrer l'existence sans pétition de principes. À la fin de son premier ouvrage, Le Traité de la Nature Humaine, il analyse la notion d'identité tant subjective qu'objective, du moi ou du réel hors de moi, comme substance sous le flux des pensées et des apparences, puis conclut qu'elle ne se ramène à aucune impression. L'identité, comme la causalité, relèvent d'une croyance imaginaire et non d'un savoir rationnel. L'habitude psychologique d'associer des idées hétérogènes nous amène à supposer l'existence permanente du réel et de soi comme même que soi, le moi. Desmond, comme Hume, doute de l'empirisme de Locke qui l'a amené à douter du bienfondé du bunker par cette démarche expérimentale: Si on n'appuie pas sur le bouton alors que se passe-t-il ? Ce qui revient à dire en bonne logique : ce n'est pas en appuyant sur le bouton qu'on vérifie que cela implique que l'on sauve le monde mais plutôt en n'appuyant pas sur le bouton. C'est une application du modus tollens: si on appuie sur le bouton et qu'il n'y a pas l'effet de destruction universelle alors appuyer sur le bouton n'est pas la cause du salut du monde. Mais, pour Hume, comme pour le philosophe éponyme, la relation d'implication étant indémontrable, cette expérimentation ne sera pas probante: qu'il ne se passe rien si on n'appuie pas sur le bouton ne prouve pas nécessairement qu'appuyer n'était pas la cause du salut du monde. Son examen des preuves lockéennes (le film de la station Perle et les relevés imprimés) lui fait inférer une hypothèse alternative: l'expérience psychologique de La Perle ne concernerait pas les observés mais les observateurs. Pas plus qu'il n'y a d'identité de l'objet observé, il n'y aurait d'identité du sujet observateur. La subjectivité du regard est plutôt relative puisque les observateurs sont eux-mêmes observables et, réciproquement, ceux qui sont observés sont susceptibles d'être observateurs. C'est ce que nous rappelions précédemment en évoquant le fait de n'être plus voyeurs mais voyants, la série nous enjoignant à avouer le caractère relatif et fini, voire relationnel et intersubjectif, de notre regard.

Ce ne sont donc pas l'étude des faits mais de la valeur morale et pratique des événements et conjonctures d'événements qui va servir d'assise aux croyances de David Hume, comme de Desmond. À la fin de la première partie du Traité sur la nature humaine, le philosophe écossais est prisonnier d'exigences contradictoires: pour réformer sa pensée et distinguer le vrai, l'illusion et l'erreur, il doit reconnaitre l'impossibilité épistémologique de cette entreprise, et vice versa. C'est la tempête mélancolique du doute ; il se demande s'il connaît quelque chose, et si cette interrogation même a un sens puisqu'il n'y a au final que des croyances. D'où son arrêt : il décide de cesser ponctuellement de spéculer pour retourner à la "vie ordinaire » et c'est dans la vie affective et morale, ayant trait à la responsabilité, la liberté, le bien et le mal et autrui, qu'il trouvera la nécessité de croire, et ce même en la spéculation. C'est la distinction du « to be » et du « ought to be » qui ouvre le dernier livre du Traité sur la nature humaine qui est reprise ici, mais radicalisée, dépouillée 
des conséquences utilitaristes qu'en tirera le penseur écossais, et greffée dans la perspective d'une métaphysique de l'Autre. La distinction n'est pas utilitaire car la série nous montre que le devoir d'appuyer sur le bouton paraît juste dans la mesure où il s'agit d'une contrainte voire d'un sacrifice qui saurait difficilement et sans hypocrisie se ramener à un calcul d'intérêt égoïste. Et, pour autant, la solution déontologique, à la Kant, selon laquelle il faut appuyer sur le bouton sans prendre en compte les conséquences particulières de l'action car c'est la seule forme de liberté rationnelle, n'est pas convaincante - si l'on exclut la question du règne des fins. Clairement, les flashbacks de Desmond, et la saison 2 dans son ensemble, nous montrent qu'appuyer sur le bouton pour appuyer sur le bouton, c'est-à-dire se résigner à un rituel creux, est aliénant et altère la personnalité. Le thème du binôme prend acte de la dimension délétère de cette attitude : donner au camarade de Desmond le nom de «Inman" (Clancy Brown) le prouve efficacement. Le nom « Inman », littéralement «l'homme intérieur », connote un rapport à l'Autre et à soi-même extrêmement ambivalent, entre la haine schizoïde (le nom évoque le double en soi, les voix qu'entend le malade mental - Desmond a la particularité de vivre ses flashbacks) et la sainteté (si l'on considère que l'expression "homme intérieur " désigne dans l'Epître de Paul aux Romains l'homme dans sa relation de ressemblance ou d'« image de Dieu» par opposition à l'« homme extérieur ", Desmond étant aussi celui pour qui l'Autre est avant tout un «Brother»).

Aussi Desmond cesse-t-il de douter du bien-fondé de l'entreprise du Cygne sur le fil. Nous sommes ici dans l'explicitation du cas de la situation éthique limite qu'est le saut de la foi que présentait la promesse de Jack à Sarah. Certes, c'est en raison de la signification morale de l'opération et des événements qu'il a vécus que Desmond confirme son attachement au fait d'appuyer sur le bouton, mais il ne le découvre qu'en aidant Locke à ne pas appuyer sur le bouton. C'est en courant le risque du scandale, en se laissant aller au doute, qu'il découvre la finalité altruiste singulière derrière un devoir qui, sinon, eût été abstrait.

D'une part, en voyant les lignes error system du 22 septembre 2004, il prend la mesure de sa responsabilité dans la catastrophe aérienne du vol Océanic 815, et ce non seulement parce qu'il était de facto absent pour rentrer les numéros à temps mais parce qu'il n'aurait pas dû être absent. La conséquence de cette fuite, et de la poursuite de son intérêt particulier au détriment de l'intérêt général, est qu'il a tué son binôme de bunker. Il comprend alors la rage de Locke puisqu'il a lui-même vécu la faute de Caïn qui est aussi celle de Jacob dans l'épisode « Accross the Sea » (S06E15), ce sacrifice de l'autre pour soi, en tuant Inman. Or cette violence fut la cause du crash, donc la genèse du récit.

Mais d'autre part, il se souvient de sa première « rencontre » avec Locke qui frappait sur la trappe, désespéré par la mort de Boone, au moment même où il voulait se suicider après avoir trouvé, entre les pages d'Un ami commun de Dickens, la lettre de Pénélope clamant sa fidélité. Locke accuse l'île de la mort de Boone car son identité consiste en un rapport, c'est-à-dire une relation à l'autre qui ne se reconnaît pas comme telle, qui se pense seule contre tous. Locke ne cesse de se définir en fonction d'une destinée lui promettant l'omnipotence qui ne peut que se muer en fatalité cruelle. Son leitmotiv, « Don't tell me what $i$ can't do! », est l'aphorisme même de l'asymétrie précédemment évoquée : il exige la présence de l'autre pour la congédier, en travaillant cependant luimême à sa propre impuissance pour restaurer une présence autre nécessairement dépréciative. Locke se définit donc contre et à partir de l'image fausse qu'il se fait de l'Autre, de l'île, de son père. En revanche, Hume s'accuse de la mort d'Inman et découvre 
la logique suicidaire animant la course qu'il a toujours mené après son honneur, la logique d'un rapport morbide à l'Autre, à l'image fausse qu'il se fait du père de sa fiancée Charles Widmore (Alan Dale), l'Autre en chef. Il le saisit lorsqu'il lit la lettre de Pénélope et prend conscience qu'il s'est lui-même exclut de la relation qu'il avait avec elle et que sa course était une fuite, ce que Charles lui avait pourtant dit et qu'il n'avait pas compris : « You just run away ». Mais, paradoxalement, cette conscience de sa faute commence déjà à le sauver, même s'il n'entend pas encore les mots de sa fiancée. La conjonction des solitudes de Locke et Hume, de ses deux orgueils impuissants, est, sur le coup, vécue comme heureuse car satisfaisant l'intérêt, voire l'amour propre, de chacun. Alors, cette conjonction pourrait indifféremment être le fruit du hasard ou du destin. Néanmoins, lorsqu'elle est remémorée, répétée dans un contexte d'extrême crise, la coïncidence de ces trajectoires associée au souvenir du meurtre d'Inman devient alors signifiante en fonction des mots de Pénélope promettant qu'elle attend Desmond, qu'elle se donne à lui et qu'il doit se rappeler qu'il est aimé. À ces mots qui seront rappelés en off, il accepte la réciprocité de la relation à l'Autre et comprend tout ce qui lui est arrivé comme le retour d'un don: «But you saved my life, brother, so that I could save yours" dit-il alors à Locke avant de se sacrifier, attribuant, par la même occasion, à la trame de la série cette signification directrice.

Cette séquence finale, comme la mort de Jack dans l'épisode «The End» (S06E17), peut s'interpréter comme une relecture du sacrifice de Spock (Leonard Nimoy) le mi-vulcain, mi-humain, de Star Trek II: The Wrath of Kahn (réal. Nicholas Meyer, 1982). Spock est le concepteur du test de Kobayashi Maru, une simulation de situation perdant-perdant où l'aspirant doit « apprendre » à perdre, accepter la nécessité de la réalité objective. Kirk (William Shatner), l'homme d'action (voire de foi puisque ce nom n'est pas sans évoquer Kierkegaard, « Kierk » signifiant " église »), est le seul à avoir réussi ce test en trichant, arguant qu'apprendre à perdre n'est pas apprendre. Il considère le réalisme de Spock et son détachement logique comme une résignation morbide. Le sacrifice de Spock est une résolution de ce dilemme. En pénétrant au cœur du réacteur nucléaire du vaisseau Enterprise pour sauver l'équipage, il se résigne au seul choix logique : la vie d'un individu compte moins que celle du Tout. Toutefois, dans le même temps, étant à moitié humain, son choix est plus que rationnel; il a une dimension affective singulière de l'ordre de la croyance, la confiance en son amitié pour Kirk. Cette comparaison nous semble justifiée car, outre les clins d'œil audiovisuels et dramatiques au film et à son remake en 2014 par le couple Abrams et Lindelof, l'oraison funèbre de Spock que récite Kirk et qui prélude à sa résurrection dans Star Trek III: The Search for Spock (réal. Leonard Nimoy, 1984) est la fin d'Un conte de deux villes (A Tale of Two Cities) de Dickens ( «e que je fais est une bien, bien meilleure chose qu'aucune que j'aie jamais faite ; le repos vers lequel je m'en vais est bien, bien meilleur qu'aucun repos que j'aie jamais connu. $\aleph^{47}$ ) qui sera le titre du premier épisode de la saison 3 de Lost.

Nous conclurons en remarquant que le problème de la croyance au monde du récit et en son ordre commun prend, dans cette saison 2, nécessairement un aspect désespérant pour susciter le saut de la foi et la possibilité de sa réitération. La promesse ne peut être entendue que par un accroissement paranoïaque du doute équivalent à la "suspension

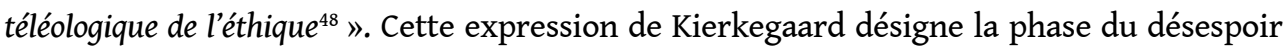
produite par la résignation au devoir qui nous paraît absurde s'il n'est répété que pour la forme. Néanmoins, c'est de cette conscience désespérée nous menant à une situation de transgression potentielle dudit devoir, que procède paradoxalement, par un effet de 
renversement dialectique, la révélation du bien fondé et de la destination transcendante de ce même devoir. D'où l'ambivalence de la réitération de l'entrée des numéros : elle a tout à la fois un aspect abstrait et aliénant mais elle est aussi libératrice. En tant que rituel, elle répète systématiquement et symboliquement l'incident dont la nature est révélée à la fin de la saison 5. L'incident est un sacrifice des autres pour soi, voire de soi contre les autres, puisque Jack lance la bombe $\mathrm{H}$ dans le site pour que le crash n'ait pas lieu et le récit non plus, privant tout le monde du spectacle de la déchéance du héros qu'il se pensait être. Appuyer sur le bouton nous permet de commémorer cette expérience originelle traumatique de passage à la limite, quand l'impossible devient possible, pour en tenir le mal à distance comme le fait le sarcophage contenant le foyer de turbulences.

Cependant on peut distinguer la répétition comme reproduction et comme recréation. Comme reproduction, l'opération est aliénante car la répétition du rituel est aussi une forme d'oubli puisqu'elle tend à se répéter pour elle-même, substituant à l'incident initial une mythologie, c'est-à-dire une téléologie rétrospective et idéologique - comme celle gouvernant la plupart des flashbacks des personnages qui se représentent leur traumatisme avec une relative complaisance égocentrique entretenant une méconnaissance d'eux-mêmes. C'est pourquoi, l'opération produit une forme de résignation dogmatique menant au doute et au désespoir qui s'en prend à cette mythologie superficielle, puis au rituel par un sacrifice de haine de l'autre pour soi, ou de soi contre l'autre, comme l'est le défi de John de ne plus appuyer sur le bouton, ou celui de Jack de faire exploser la bombe $\mathrm{H}$ dans l'épisode «The Incident » (S05E16-17). La répétition révèle alors que ces personnages tendent à la fin, la fin de l'autre, la leur, celle du récit; ils veulent l'apocalypse dans le sens qu'on lui prête d'ordinaire, celui de catastrophe, de violence transcendant toute raison ${ }^{49}$. Et pourtant, à l'acmé de la crise, l'opération retrouve un sens originel, celui de la promesse derrière l'incident, derrière le sarcophage. Le doute que produit la répétition de l'opération devient une révélation pour Desmond, via la patience de Pénélope. Cette révélation, qui est le sens littéral du terme " apocalypse », est que la seule réponse à cette situation est le sacrifice de soi pour l'autre qui est cette transcendance, cet au-delà du récit sur lequel repose le récit. Ce sacrifice est figuré par l'ouverture du cercueil qu'est le Cygne. Or ce cercueil est la promesse de Lost, celui de Christian qui reste ouvert et vide près de la source et des dépouilles « d'Adam et Eve » dans l'épisode "White Rabbit» (S01E05) et autour duquel Christian se réconcilie avec son fils et réunit les rescapés à la fin de la série. C'est pour cela que Desmond a la clé, voire est la clé de la série et que son régime de flashbacks est unique : il ne se représente pas mais vit son passé, étant réellement présent à lui-même ${ }^{50}$. Ainsi la répétition ouvre-telle la voie à une recréation et devient-elle proprement religio-dans le sens de l'étymologie contestée de Lactance, cette fois, c'est-à-dire religare ce qui relie les hommes entre eux et lie l'immanent au transcendant ${ }^{51}$. Elle fait advenir un monde commun à tous les milieux individuels, une unité simultanée d'un univers de coexistence des sujets conscients en ouvrant le cercueil.

Explicitant l'expérience de Jack de l'épisode « Man of science, Man of faith », la leçon que nous donne Desmond Hume, à la fin de la saison, est donc que cette instance transcendante est autrui et qu'il nous est proche dans son infinie distance. L'important n'est donc pas tant en quoi, mais en qui, je veux croire, c'est-à-dire à l'autre dans sa différence, sa patience, sa proximité lointaine, comme l'atteste la fin de l'épisode «Live together, Die alone ». En se sacrifiant pour faire sauter la station du Cygne, Desmond fait exister une autre dimension - le premier et seul contrechamp, voire hors-champ, à l'île, 
qui ne soit ni souvenir, ni flashforward ni flash-sideways, mais est contemporain des aventures des survivants, et signe d'une coprésence, celle en qui croire, Penelope.

\section{BIBLIOGRAPHY}

BATESON Gregory, Vers une Ecologie de l'Esprit 2, Paris, Seuil, 2008.

BENVENISTE Emile, Le Vocabulaire des institutions Indo-Européennes : Pouvoir, Droit, Religion, Paris, Éditions de Minuit, 1969.

BORTZMEYER Gabriel, « Serial Cruiser », Trafic n90, Paris, POL, 2014, p.45-58.

CICERON, La Nature des dieux, Paris, Les Belles Lettres, 2002.

COLERIDGE Samuel, La Ballade du vieux marin et autres textes, Paris, Gallimard, 2007.

DICKENS Charles, Un Conte de deux villes, Paris, Folio, 1989.

DOSTOÏEVSKI Fiodor, Les Carnets du sous-sol, Arles, Actes Sud, 1992.

_-_. Les Frères Karamazov, Paris, Folio, 1994.

ECO Umberto, De Superman au Surhomme, Paris, Le Livre de Poche, 1995.

GIRARD René, Mensonge romantique et Vérité romanesque, Paris, Pluriel, 2010.

HATCHUEL Sarah, Lost : Fiction vitale, Paris, Presses universitaires de France, 2013.

HUME David, Système sceptique et autres systèmes, Paris, Seuil, 2002.

IRWIN William et Kaye SHARON, Lost and Philosophy: Think together, Die alone, Hoboken, John Wiley and Sons, 2010.

KIERKEGAARD Søren, La Reprise, Paris, Garnier-Flammarion, 1990.

_-_. Crainte et Tremblement, Paris, Payot-Rivages, 1999.

_-_. Le Traité du désespoir, Paris, Folio, 1988.

LACTANCE, Institutions divines (Livre IV), Paris, Cerf, 1992.

LÉVINAS Emmanuel, Entre nous, Paris, Le Livre de Poche, 1990.

MARION Jean-Luc, La Croisée du visible, Paris, Presses Universitaires de France, 2007.

PASCAL Blaise, Pensées, Paris, Folio, 2004.

THIELLEMENT Pacôme, Les mêmes yeux que Lost, Paris, Léo Scheer, 2011.

TODOROV Tzvetan, Introduction à la littérature fantastique, Paris, Seuil, 1976.

WEINRICH Harald, Le Temps, Paris, Seuil, 1973.

\section{NOTES}

1. David Hume, Système sceptique et autres systèmes, Paris, Seuil, 2002, p. 251. 
2. Cf. édition DVD de Lost, Saison 2, Bonus. Pacôme Thiellement montre que ce syndrome du Petit Prince et du Chat de Schrödinger, de la boîte où tout désir d'ailleurs et de possibles est comblé sans prise en compte d'un quelconque principe de contradiction (sinon sa localisation), est repris par Cuse et Lindelof qui œuvreraient à « refermer les possibles ». Voir Pacôme Thiellement, Les mêmes yeux que Lost, Paris, Léo Scheer, 2011, p. 10-14. Il faut faire le deuil d'attentes démesurées certes, et cependant nous ajouterons que ce deuil a comme effet paradoxal un appel à une réouverture de la promesse de récit.

3. Roman que lisent Hurley (Jorge Garcia) et Sawyer (Josh Holloway) dans cette même saison (épisode 20, «Two for the road ») et que brûlent Jack (Matthew Fox) et Locke (Terry O'Quinn).

4. Cf. Cicéron, De la Nature des dieux, Paris, Les Belles Lettres, 2002, p. 88-90. Benveniste, Le Vocabulaire des institutions Indo-Européennes : Pouvoir, Droit, Religion, Paris, Éditions de Minuit, 1969, p. 265.

5. Comme le sont les membres de la Dharma Initiative, les initiés s'opposant aux néophytes en prétendant saisir ce qu'il y a à l'initial, à l'origine, voire au fondement de l'univers du récit, sans apercevoir, sans doute, que Dharma Initiative a, dans les termes, tout de l'oxymore. Si le Dharma est la roue et le mouvement éternel du Destin chez les Hindous, l'Eternel Retour, comment peutelle avoir accès à l'avènementiel, à l'irruption du nouveau qu'est l'initiative?

6. D'une certaine façon les spectateurs sont déjà prévenus du non-retour sur investissement de leur crédit : ils regardent une série qui s'appelle Lost - encore faut-il comprendre que c'est perdu pour perdu.

7. Le MacGuffin est une technique d'écriture développée, entre autre, par Hitchcock, qui consiste à présenter une intrigue-prétexte afin de mettre en image le renversement des rapports sentimentaux entre les personnages, comme l'histoire de l'uranium caché dans la cave à vin dans Notorious (Alfred Hitchcock, 1946), de la machine de Rambaldi dans Alias, ou de la «patte de lapin » dans Mission Impossible III (J.J. Abrams, 2006). Voir Alfred Hitchcock et François Truffaut, Hitchcock / Truffaut, Paris, Gallimard, 2003, p.111-113 et p.139-144.

8. Voir Fiodor Dostoïevski, Les Frères Karamazov, Paris, Folio, 1994, p. 345-368.

9. Voir Thiellement, p. 99-104.

10. The willing suspension of disbelief est selon le poète anglais Samuel Coleridge, dans Biographia Litteraria, la condition à laquelle le récepteur consent à croire en la fiction racontée et suspend son doute. C'est cependant une croyance sous condition supposant des conventions sociales, des codes culturels d'entrée et de sortie du récit. Voir Samuel Coleridge, La Ballade du vieux marin et autres textes, Paris, Gallimard, 2007.

11. Voir Umberto Eco, De Superman au Surhomme, Paris, Le Livre de Poche, 1995, p. 27-34 et p. 113-45.

12. Voir Gabriel Bortzmeyer, «Serial Cruiser », Trafic n 90, Paris, P.O.L., 2014, p. 45-58.

13. Voir Bortzmeyer, op.cit. p. 49.

14. Voir Sarah Hatchuel, Lost : Fiction vitale, Paris, Presses universitaires de France, 2013, p. 34-39.

15. Comme nous le verrons, ces thèmes de la foi et du sacrifice se retrouvent particulièrement dans une autre collaboration de Lindelof et Abrams, Star Trek: Into Darkness (réal. J. J. Abrams, 2013) dont la séquence du sacrifice de Kirk (Chris Pine) est une variation lostéeenne autour de Star Trek II: The Wrath of Kahn (réal. Nicholas Meyer, 1982).

16. Voir Pascal, Pensées, Paris, Folio, 2004, p. 186. Cette problématique, aussi vieille que la question de la distinction entre l'idole et l'icône, est aussi celle de l'irreprésentable. Cette question anime l'art baroque, comme nous le verrons, et traverse aussi la filmographie de Spielberg, influence avouée d'Abrams. Ainsi dans Indiana Jones et la Dernière Croisade, Spielberg figure-t-il élégamment le saut de la foi : le personnage se jette dans un gouffre et est soutenu par un pont en trompe-l'œil dans la fiction mais dont le réalisateur révèle la présence au spectateur, non par une prise directe, mais par le biais d'un effet visuel. 
17. Le thème du sacrifice est ce qui lie explicitement Desmond et Locke. La mort de Boone (Ian Somerhalder), fils spirituel de Locke, est ce qui amènera Desmond à lui envoyer un signe de sa présence dans l'épisode « Deus ex Machina ». Sachant que Locke lui-même fit don d'un rein pour son père qui le trahit ensuite.

18. Kierkegaard, Crainte et Tremblement, Paris, Payot \& Rivages, 2000.

19. Voir Dostoïevski, p. 61.

20. Voir Sander Lee, «See you in another life, Brother: Bad faith and authenticity in three Lost souls", in William Irwin and Sharon Kaye, LOST and Philosophy: Think together, Die alone, John Wiley and Sons, Hoboken, 2010, p. 120-41.

21. Voir Hatchuel, p. 34-9.

22. Voir Platon, Théétète, 201c-210a, Paris, Garnier Flammarion, 1994, p. 277-303.

23. Kierkegaard, Traité du Désespoir, Paris, Folio, 1988, p. 104.

24. Le philosophe danois utilise également les termes de paradoxe voire de scandale (ce dernier ayant alors un sens évangélique et théologique).

25. La référence à l'œuvre de Dostoïevski à l'aune de l'envie est explicite puisque Locke donne à celui qui se fait appeler Henri Gale, l'ouvrage Les Frères Karamazov, tout en commentant ce choix en évoquant la jalousie profonde de Hemingway pour l'auteur russe, dans l'épisode « Maternity Leave » (S02E15).

26. «La foi est justement ce paradoxe : l'individu, en tant qu'individu, est plus grand que le général, il est justifié devant le général, il ne lui est pas subordonné, mais lui est supérieur, et il l'est de telle manière, observez-le bien, que c'est l'individu qui, après avoir été subordonné en tant qu'individu au général, devient, à travers le général, l'individu dont l'individualité est supérieure au général, parce que l'individu, en tant qu'individu, est en rapport absolu avec l'absolu. », Søren Kierkegaard, Crainte et Tremblement, Paris, Payot-Rivages, 1999, p. 110.

27. Emmanuel Lévinas, Entre nous, Paris, Le Livre de Poche, 2004, p. 143-64.

28. «Mais on est curieux du résultat comme on l'est de la conclusion d'un livre; de l'angoisse, de la misère, du paradoxe on ne veut point entendre parler. En esthète, on fait la cour au résultat et il arrive à l'improviste, et tout aussi facilement, qu'un prix à la loterie et, lorsqu'on l'a entendu on demeure édifié. » Kierkegaard, p. 121.

29. D'ailleurs il considère que ce qui rapproche le plus de cette écriture répétant le «saut » dans sa contradiction intime d'un point de vue profane est l'œuvre de Shakespeare, ce qui est confirmé par les références de la saison 2 (voir plus loin). « Je ne sais, ami lecteur, ce que tu as pu faire dans la vie, mais maintenant efforce ton cerveau, rejette tout faux-semblant, pour une fois avance à découvert, dénude ton sentiment jusque dans ses viscères, abats toutes les murailles qui séparent d'ordinaire le lecteur de son livre et lis alors Shakespeare... tu verras des conflits qui te feront frissonner. » Kierkegaard, Traité du Désespoir, Paris, Folio, 1988, p. 244.

30. Voir Kierkegaard, La Reprise, Paris, Garnier-Flammarion, 1990. Comme pour Jack, John et Desmond, ce problème de la répétition apparaît à l'occasion d'une rupture avec sa fiancée, Régine Olsen et pour la même raison officielle qu'eux, un péché du père. Lors de la tentative ratée de renouement, interprétée à l'aune d'une exégèse du Livre de Job, Kierkegaard comprend que l'impossibilité de reproduire et modifier ce qui s'est passé, de reprendre la possibilité d'autrefois aujourd'hui perdue et du refus de se résigner à une fausse culpabilité ou à une accusation de l'autre désespérante, naît la possibilité paradoxale, la pure possibilité d'une répétition de ce que promettait leur union, une relation réciproque. C'est pourquoi il fallait que Kierkegaard soit séparé de Régine pour pouvoir être tout à fait avec elle, idem pour Desmond et Pénélope à la fin de la saison.

31. L'interprétation de Pacôme Thiellement selon laquelle Lost est une série sur le malaise de l'homme moderne, du ressentiment, est corroborée par l'allusion du récit à Dostoïevski. En revanche, faire des pères les responsables de ce ressentiment est un peu réducteur car la série condamne les personnages parricides et se clôt tout de même sur une réconciliation entre Jack et 
Christian. D'autre part, dans l'épisode « The Incident » (S05E17), nous apprenons que l'histoire de sa première opération dont Jack tire orgueil depuis le pilote de la série et qui revient comme un leitmotiv pour montrer l'intimité de son lien avec Kate, est un mensonge : c'est son père qui lui dit de respirer et de compter jusqu'à cinq, et l'on peut même se demander si ce dernier n'a pas fini l'opération à la place de son fils.

32. Voir Pacôme Thiellement, p. 31.

33. Les configurations temporelles du récit rendent cette tonalité affective de ressentiment, voire la dialectique de la confiance et de la défiance. Les flashbacks, flashforwards, la dualité temporelle 1977/2008 et les flash-sideways produisent des effets d'écriture brillants qui contribuent à désorienter le spectateur et donc à rendre incertain le présent de l'île. Mais d'une part les flashforwards ne sont pas une vraie ouverture au futur, seule la fin de la série pourrait l'être, car, comme le soutient Weinrich, le temps de la narration est toujours celui du passé, de la rétrodiction. Or Lost en prend tout à fait acte. Ces variations se font sur le mode de la répétition, comme l'est l'entrée des numéros dans le bunker. Elles révèlent une profonde vanité car elles se réfèrent toujours au présent de l'île que refusent de vivre la plupart des personnages de la série et qui fait de leur présent passé ou de leur présent futur passé des regrets voire des remords. Ce refus est tel qu'il mène à la destruction de l'île dans les flash-sideways avec le paradoxe que celleci existe toujours pourtant par ailleurs. Voir Harald Weinrich, Le Temps, Paris, Seuil, 1973.

34. Voir Dostoïevski, Les Carnets du Sous-Sol, Paris, Actes Sud, 1992, p. 37.

35. Initialement, l'épisode aurait dû être réalisé par Darren Aronofsky. Convoquer le réalisateur de $\Pi$ (1998) tendrait à prouver la place majeure qu'occupe cet épisode dans la série et sur le thème croyance et savoir, mais le réalisateur n'aurait pu se rendre à Hawaï car sa compagne Rachel Weizs était alors enceinte. Sans doute une femme enceinte ne peut-elle se rendre sur l'île - ou quand la réalité rejoint la fiction...

36. Ce lieu est reconnu comme étant le lieu d'un sacrifice, celui de Boone dans l'épisode «Deus ex machina " (S01E19), et le mémorial de Yemi (Adetokumboh McCormack) dans « The $23^{\text {rd }}$ Psalm » (S02E10).

37. Voir Tzvetan Todorov, Introduction à la littérature fantastique, Paris, Seuil, 1976, p. 46-96.

38. On précisera qu'à la fin de la saison 2, Sawyer (Josh Holloway), personnage éminemment manipulateur, ne cesse de répéter le verbe to screw avec ce double sens de «baiser " et de «se faire avoir " à propos d'Ana Lucia (Michele Rodriguez) et de "Henry "/Ben (Michael Emerson) dans les épisodes 22 ( Three minutes »), et 23-24 («Live together, Die Alone »).

39. Voir Gregory Bateson, Vers une Ecologie de l'esprit II, Paris, Seuil, 2008, p. 9-105.

40. La référence à cet épisode est d'autant plus confirmée que, selon Pacôme Thiellement, la Perle fait allusion à la parabole du prince à la perle dans l'Évangile apocryphe de Thomas - récit qui sert de trame au film Knight of Cups (Terence Malick, 2015).

41. Voir l'article de Sarah Hatchuel et Randy Laist dans ce numéro.

42. Voir Thiellement, p. 114-16.

43. Voir Jean-Luc Marion, La Croisée des Visibles, Paris, Presses Universitaires de France, 2005, p. 83-115.

44. Voir René Girard, Mensonge romantique et Vérité romanesque, Paris, Pluriel, 2010, p.40-51 et p. 226-32. L'huître perlière comme œil critique omnipotent, et le cygne, modèle intouchable, sont deux figures proustiennes que présente Girard dans son analyse de À la recherche du temps perdu. Elles ont en commun d'être des illusions reposant sur le refus d'admettre le caractère intersubjectif du désir et donc de se méprendre sur la valeur de l'objet désiré, méprise dont le personnage de Swann sera le premier révélateur pour le narrateur.

45. Lindelof reconnaissant l'influence de la trilogie Indiana Jones de Steven Spielberg et George Lucas sur l'écriture de Lost, on peut se demander dans quelle mesure l'énoncé « let go. » n'est pas influencé par La Dernière croisade (The Last Crusade, 1989). Il s'agit de la réplique de Henry Jones (Sean Connery) à son fils (Harrison Ford) lorsqu'il le nomme « Indiana » pour la première fois et 
non plus « Junior » et l'adjure de renoncer au Graal. C'est en renonçant au prestige du Graal qu'il en reçoit l'illumination et non en en faisant une récompense à l'instar de l'archéologue nazie Elsa Schneider (Alison Doody).

46. Emmanuel Lévinas, Entre nous, Paris, Le Livre de Poche, p. 212.

47. Charles Dickens, Un Conte de deux villes, Paris, Folio, 1989, p. 400.

48. Voir Kierkegaard, Crainte et Tremblement, Paris, Payot-Rivages, 1999, p. 107-25.

49. « The Incident » est d'ailleurs doublement apocalyptique puisque l'île est menacée par Daniel (Jeremy Davies) via Jack en 1977 et par l'innommable homme en noir via John en 2007, Daniel et John (Jean) étant les prénoms d'auteurs de livres dit « apocalyptiques » dans l'Ancien et Nouveau Testaments.

50. Lorsqu'il a des visions du futur dans la saison 3, elles ne le concernent pas lui mais Charlie (Dominic Monaghan) et l'enjeu de ces flashforwards, annonçant ceux de la saison 4, est d'accompagner Charlie dans l'acceptation de la possibilité de mourir pour sauver Claire (Emilie de Ravin) et Aaron. Celui-ci mourra d'ailleurs en parlant à Penny dans «Through the Looking Glass » (S03E24), ce qui confirme l'influence médiatrice de Charles Dickens entre Desmond et Penny puisque c'est un trio (une trinité ?) de Charles qui structure leur relation : le père (Charles Widmore), le fils que Desmond a avec Penny (Charlie Hume) et l'esprit de sacrifice (Charlie Pace). 51. Voir Lactance, Institutions divines, Livre IV, Paris, Cerf, 1992, p. 233-37.

\section{ABSTRACTS}

This essay deals with the themes of the leap of faith, the ritual and the sacrifice in the television show Lost, concentrating mainly on season 2. It questions Lost's promise to spectator as far as narration is concerned. This promise seems to be paradoxical: the story requires the viewer to be suspicious and even to distrust what s/he sees. Our work examines the Swan Station apparatus and the opposition between belief and knowledge which is reflected in the antagonism between Jack Sheppard and John Locke and finds a solution in the sacrifice of Desmond Hume. The essay analyses the episodes "Man of science, Man of faith" (S02E01), "?" (S02E21) and "Live together, Die alone" (S02E23-24). The first episode is interpreted in relation to Kierkegaard's works to show that belief and knowledge are two phases before a character's leap of faith, which consists in being open to others. However, the repetition of this leap is problematic; the second episode is understood as a focalisation on the despair of one character, Locke, who gives us a reflexion on the way to look at the TV show as an undecidable oscillation between distrust and trust, i.e. between the Pearl and the Swan. The third one, compared with David Hume's writings and to Star Trek II: The Wrath of Kahn, allows to reveal the solution offered by the show, which would be selfsacrifice, mainly the sacrifice of one's own pride, evident in Desmond and Penelope's relationship.

Notre article étudie les thèmes du saut de la foi, du rituel et du sacrifice dans la série Lost en interrogeant surtout la saison 2. Par voie de réflexion, il interroge la promesse de Lost, c'est-àdire le type de crédit que le récit prescrit au spectateur d'accorder à ce qui est raconté. La promesse paraît paradoxale, le récit exigeant de se méfier voire de se défier pour mieux croire. Notre travail examine à cet effet le dispositif de la Station du Cygne et l'opposition entre croyance et savoir paraissant se cristalliser dans l'antagonisme de Jack Shephard et John Locke et se résolvant dans le sacrifice d'une figure intermédiaire, Desmond Hume. Il analyse donc les 
épisodes « Man of science, Man of faith » (S02E01), «?» (S02E21) et «Live together, Die alone » (S02E23-24). Le premier épisode est interprété à l'aune de l'œuvre de Kierkegaard pour montrer que croyance et savoir sont deux phases pour un même personnage vers le saut de la foi qui consiste dans l'ouverture à autrui. Cependant la répétition de ce saut est problématique. Ainsi le deuxième est compris comme une focalisation sur le désespoir d'un personnage, Locke, offrant une réflexion sur la manière de voir la série comme une oscillation indécidable entre méfiance et confiance, la Perle et le Cygne. Le troisième, comparé aux écrits de David Hume et au film Star Trek II: The Wrath of Khan, permet d'exposer la solution que propose la série, qui serait le sacrifice de soi (et surtout de son orgueil) pour l'autre, manifeste dans la relation de Desmond à Penelope.

\section{INDEX}

Mots-clés: Lost, suspension volontaire d'incrédulité, scepticisme, foi (acte de), sacrifice, répétition, baroque, Hume David, Kierkegaard Søren, Dostoïevski Fiodor

Keywords: suspension of disbelief, skepticism, leap of faith

\section{AUTHOR}

\section{GUILLAUME DULONG}

Guillaume Dulong, agrégé de philosophie et docteur en études cinématographiques, est professeur de philosophie et de cinéma en lycée, ainsi que chargé de cours en cinéma à l'université Michel de Montaigne Bordeaux III. S'inspirant des théories d'André Gaudreault et Tom Gunning, son travail de thèse a porté sur le genre cinématographique de l'imaginaire et l'influence des technologies numériques sur les modes de narration contemporain de fantasy. Guillaume Dulong, who holds an 'agrégation' in philosophy and a PD in film studies, is a professor of philosophy and cinema in high school; he also teaches cinema at the University of Bordeaux Michel de Montaigne. Inspired by the theories of André Gaudreault and of Tom Gunning, his PhD thesis explored the filmic genre of fantasy and the influence of digital technologies on contemporary narration. 